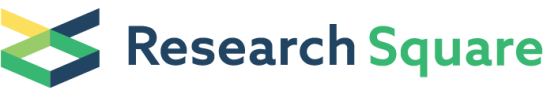 \\ Preprints are preliminary reports that have not undergone peer review. \\ They should not be considered conclusive, used to inform clinical practice, or referenced by the media as validated information.
}

\section{Improving the efficiency of nursing work for patients with severe COVID-19}

\section{Mu Zhihui}

Department of Critical Care Medicine, Wuhan Houshenshan Hospital; Department of Neurosurgery, First Affiliated Hospital of PLA Air Force Military Medical University

\section{Luo Meimei}

Department of Orthopedics, First Affiliated Hospital of PLA Air Force Military Medical University

\section{Zhong Ya}

Department of Critical Care Medicin, Wuhan Huoshenshan Hospital; Dialysis Center of the First Affiliated Hospital for PLA Air Force Military Medical University

\section{Li Min}

Department of Critical Care Medicine, Wuhan Huoshenshan Hospital; Transplant Center, First Affiliated Hospital of PLA Air Force Military Medical University

\section{Li Minghui}

Department of Orthopedics, First Affiliated Hospital of PLA Air Force Military Medical University

\section{Wang Yu}

Department of Neurosurgery, First Affiliated Hospital of PLA Air Force Military Medical University

\section{Nan Zhang (D 541967734@qq.com)}

Department of Critical Care Medicine, Wuhan Houshenshan Hospital

\section{Research article}

Keywords: Novel Coronavirus Pneumonia, Nursing, Working efficacy

Posted Date: September 16th, 2020

DOI: https://doi.org/10.21203/rs.3.rs-64805/v1

License: (a) (i) This work is licensed under a Creative Commons Attribution 4.0 International License. Read Full License 


\section{Abstract \\ Background}

Exploring new ways to improve the efficiency of nursing work for patients with severe new coronavirus pneumonia.

\section{Methods}

A total of 372 clinical nursing shifts from February 9, 2020 to April 11, 2020 were analyzed in this study. Shifts were divided into a control group (186 shifts before reorganization) and an observation group (186 shifts after reorganization). Following improvements were applied: wearing the protective equipment, communication between inside and outside the contaminated area, and time needed to restock the bedside consumables.

\section{Results}

After the new method was applied, changing of protective equipment during worktime was reduced (5\% $(9 / 186)$ in the observation group vs. $15 \%(27 / 186)$ in the control group; $\mathrm{P}=0.003)$. Moreover, the time needed to transfer items between inside and outside contaminated area and time for replenishment consumables for bedside treatment was shorter in the observation group compared to the control group $(1.98 \pm 1.41,6.86 \pm 2.25$ vs. $2.52 \pm 1.97,10.81 \pm 4.45$, respectively; all $\mathrm{P}<0.002)$.

\section{Conclusion}

The new applied measures have improved the nursing efficiency in patients with severe novel coronavirus pneumonia.

\section{Background}

The novel coronavirus pneumonia (COVID-19) is an infectious disease caused by a newly discovered coronavirus that is rapidly spreading worldwide. As of July 18,2020 , COVID-19 has infected more than 14 million people and killed 597 thousand, through 216 countries and regions [1]. Wuhan Huoshenshan Hospital was established as the first batch of new coronary pneumonia specialist hospitals and achieved initial results.Due to the rapid progress of the disease, some patients with COVID-19 develop severe symptoms, such as, as severe acute respiratory distress syndrome or even shock, combined with multiple organ failure [2, 3]. For COVID-19 patients, medical personnel monitor vital signs, consciousness, blood oxygen saturation, and assess the function of systemic organs $[4,5]$. However, due to the characteristics of the disease and working environment and other factors, nursing work is challenging [6-8]. Thus, it is urgent to summarize the experience and formulate specific implementation strategies to improve work 
efficiency. In this study, we established and validated a new plan for improving the efficiency of nursing work for patients with severe COVID-19.

\section{Methods}

\section{General information}

A total of 372 clinical shifts (Huoshenshan Hospital, Wuhan, Hubei, China) were monitored from February 9, 2020 to April 11, 2020, during which 62 COVID-19 patients were treated. Huoshenshan Hospital is an emergency hospital, built between 23 January and 2 February 2020, in response to the COVID-19 pandemic.

The study was approved by the ethics committee of First Affiliated Hospital of PLA Air Force Military Medical University. All participants provided written informed consent.

\section{Grouping}

Shifts were divided into a control group (186 shifts) and an observation group (186 shifts). The 186 shifts before the rectification were set as the control group; during this shift, a routine nursing mode was adopted. In the observation group, the wearing comfort of protective equipment was improved to reduce the frequency of changing the protective equipment. Also, the transfer of articles in the contaminated area was standardized.

\section{Observation index}

The monitoring process included: 1 ) changing protective equipment during worktime; 2) communication between inside and outside the contaminated area; 3 ) time needed to restock the bedside consumables [9].

\section{Rectification measures}

\section{Wearing protective equipment}

The following improvements were made: $\otimes$ the hand sanitizer, laundry fluid, iodophor, or mist spray were evenly applied to goggles, leaving the liquid to dry. It was ensured that the edge of the mask fits between the upper edge of the mask and the skin (from the lower part of the outer eyelid through the bridge of the nose to the lower part of the opposite eyelid). In addition, the positive pressure tightness test and the negative pressure tightness test [5] were performed. During the process, if the airflow passed through the upper edge of the mask, the mask needed to be adjusted. If there was a visible gap, the mask could be worn after the application of facial decompression paste. The fixation position of the frenulum can be slightly close to the top of the head, so there is a certain pressure between the upper edge of the mask and the skin, and fit as closely as possible. $\llbracket$ Goggles, shoe cover, and other ties were adjusted according to personal conditions and properly tightened. If the goggles are too tight, headache and vomiting may 
occur; if the shoe cover is too tight, numbness of lower limbs may occur. 区After wearing the mask, it was necessary to check whether the fixation was firm so as to prevent the frenulum from slipping forward from the head, affecting the sealing effect, or sliding backward, thus making the frenulum press on the upper part of both ears, aggravating already uncomfortable experience and even causing skin ulceration. When wearing the goggles, they can easily slide down, especially after engaging in the activities, so it was ensured that the lower edge of the inner mask was close to the nasal wing, which affects the breathing. Therefore, the position of the goggles close to the nasal bridge should not be too low. $\mathbb{Q}$

\section{A delivery system for contaminated areas}

The following improvements were made: 区the items were fixed, classified, and clearly marked. It was established that a network communication platform, such as WeChat working group would be useful. Publish the item placement diagram on the WeChat working group so that all medical staff can grasp it.After use, they should return to their original position according to the requirements and hand over strictly. For each shift, the designated person in charge shall count all the items consumed and supplemented for each shift and send them to the working group at least 30 minutes before the shift handover. The next shift checked the information before entering the contaminated area, and the required items were brought into the contaminated area through the warehouse of the clean area. $\otimes_{T}$ The reserve base was adjusted according to the consumption reported by each shift every week, the warehouse was timely notified of insufficient surplus items and substitutes were requested. 《Before entering the work area, the basic information of the patients were shared in advance via computer in the cleaning area, and the doctors and nurses jointly pre-judged the required items. $\otimes T$ The address book of each ward was created, keeping the communication smooth, and making emergency deployment under special circumstances. Meanwhile, this reduced the communication time between inside and outside the contaminated area and reduced the waiting time of goods delivery.

\section{Establishing a new system for the use of consumables}

The following improvements were made: $₫$ The configuration of the treatment vehicle was standardized. Every three beds were equipped with a treatment vehicle, and the management was fixed. The type and quantity of consumables stored in the treatment vehicle were standardized, and previous staff members supplemented replacement consumption. According to the workflow and reasonable treatment of patients, the successor estimated the number of consumables needed on duty, to reduce the time of repeated replenishment of consumables and the time needed to enter and leave the treatment room. \Modular first-aid supplies kit group was set up including emergency airway management and peripheral venous catheterization, etc. For example, oxygen mask, high flow oxygen nasal catheter, endotracheal intubation, guidewire, disposable laryngoscope, noninvasive ventilator mask, disposable ventilator humidification tank, ventilator pipe, etc. were prepared in the emergency airway treatment box group, and the consumables for each group were prepared in 3 sets. $\otimes$ Spare bed management. After patients were transferred out, when the beds were temporarily empty, the person on duty prepared a base number of conventional consumables for new patients, including oxygen inhalation devices, electrode piece, 
ventilator pipeline, etc. $\otimes$ Strengthening information communication. When it was determined after a consultation that the transferred or newly admitted patients from the ward were confirmed by the consulting doctor and the department of critical care medicine, the basic information of the patients and the special materials to be prepared were also communicated at the same time, and the nurses made preparations in advance.

\section{Statistical analysis}

SPSS 22.0 software was used for statistical analysis, measurement data were expressed as mean \pm standard deviation, and a t-test was performed. Count data were tested by the $\chi^{2}$ test. A P $<0.05$ was considered statistically significant.

\section{Results}

\section{Comparison of general data of patients treated in two shifts}

Twenty-five COVID-19 patients were treated in the first 186 shifts (control group), and 37 cases were treated after refinement (observation group). As shown in Table 1, there were no significant differences in disease classification, gender, age, disease condition, basic diseases between the two groups $(P>0.05)$. 
Table 1

Clinical presentations of COVID-19 patients in 2 groups

\begin{tabular}{|c|c|c|c|c|}
\hline \multirow[t]{2}{*}{ Parameter } & Total & Control group & Observation group & $P$ value \\
\hline & $N=62$ & $N=25$ & $N=37$ & \\
\hline Types n(\%) & & & & 0.91 \\
\hline Severe & $28(45)$ & $12(48)$ & $16(43)$ & \\
\hline Critical & $34(55)$ & $13(52)$ & $21(57)$ & \\
\hline Gender n (\%) & & & & 0.93 \\
\hline Female & 19(31) & $8(32)$ & $11(30)$ & \\
\hline Male & $43(69)$ & $17(68)$ & $26(70)$ & \\
\hline Age (year), M (QL, QU) & $69(63,78)$ & $69(63,73)$ & $71(62,81)$ & 0.36 \\
\hline Acute respiratory failure $\mathrm{n}(\%)$ & & & & 0.93 \\
\hline No & $48(77)$ & 19(76) & $29(78)$ & \\
\hline Yes & $14(23)$ & $6(24)$ & $8(22)$ & \\
\hline Other organ failure n (\%) & & & & 0.34 \\
\hline No & $42(68)$ & $16(64)$ & $26(70)$ & \\
\hline Yes & $20(32)$ & $9(36)$ & $11(30)$ & \\
\hline Basic disease n (\%) & & & & 0.92 \\
\hline No & $9(15)$ & $4(16)$ & $5(14)$ & \\
\hline Yes & $53(85)$ & $21(84)$ & $32(86)$ & \\
\hline
\end{tabular}

\section{Replacement of protective equipment during worktime}

After the new method was applied, $5 \%(9 / 186)$ of nurses changed protective equipment during work time compared to $15 \%(27 / 186)$ in the control group, and the differences were statistically significant $(\mathrm{x} 2=8.89, \mathrm{P}=0.003 ;$ Table 2$)$.

Table 2. Comparison of protective equipment replacement during worktime (shift) 


\begin{tabular}{|llll|}
\hline Group & Case & Incidence (\%) & Non-incidence (\%) \\
\hline Control & 186 & $27(15 \%)$ & $159(85 \%)$ \\
\hline Observation & 186 & $9(5 \%)$ & $177(95 \%)$ \\
\hline$\chi^{2}$ & & & 8.89 \\
\hline$P$ & & & 0.003 \\
\hline
\end{tabular}

Time for transferring items between inside and outside of the contaminated area

The time for transferring items between inside and outside of contaminating area was shorter in the observation group $(1.98 \pm 1.41)$ compared to the control group $(2.52 \pm 1.97)$; the difference was statistically significant ( $t=3.04, P=0.002 ;$ Table 3 )

Table 3. Time for transferring items between inside and outside of contaminating area (mean \pm standard deviation)

\begin{tabular}{|lll|}
\hline Group & Case & Times of establishing links \\
\hline Control & 186 & $2.52 \pm 1.97$ \\
\hline Observation & 186 & $1.98 \pm 1.41$ \\
\hline $\mathrm{t}$ & & 3.04 \\
$\mathrm{P}$ & & 0.002 \\
\hline
\end{tabular}

\section{Time for replenishment of consumables}

The time required for replacement of bedside consumables was shorter in the observation group (6.86 \pm 2.25 times) compared to the control group (10.81 \pm 4.45$)$; the difference between the two groups was statistically significant. ( $t=10.80, P<0.001)$. (Table 4).

Table 4. Time for consumables replenishment (mean \pm standard deviation)

\begin{tabular}{|lll|}
\hline Group & Case & Average number of round trips \\
\hline Control & 186 & $10.81 \pm 4.45$ \\
\hline Observation & 186 & $6.86 \pm 2.25$ \\
\hline $\mathrm{t}$ & & 10.80 \\
\hline $\mathrm{P}$ & & $<0.001$ \\
\hline
\end{tabular}

\section{Discussion}


During the epidemic period, especially in the early stage of the epidemic, infection rates, and deaths among healthcare staff significantly increased[10,11]. The main nursing work of the ICU during novel coronavirus pneumonia is to implement treatment, life care, and psychological nursing for patients with confirmed infection [12-16]. In addition, the nursing work includes cleaning and disinfection of the ward environment, waste sorting and recycling, and in-hospital control $[17,18]$. Thus, strengthening the protection of medical staff during the epidemic is of crucial importance [19,20]. In this study, we established and validated a new plan for improving the efficiency of nursing work for patients with severe COVID-19.

\section{Reducing protective equipment discomfort}

Some studies have shown that the fogging of goggles is a common problem when caring for COVID-19 patients [21]. The incidence of fogging of goggles within two hours after entering the contaminated area is approx. 48.3\% [22]. In the present study, we found that the incidence of dizziness, headache, vomiting, and other symptoms were $55 \%$; these conditions were associated with tightening the goggle's fixation belt. Moreover, $15 \%$ of nurses were required to change the protective equipment after entering the infected area, and the average time for entering and leaving the contaminated area was 35 minutes. The main causes for leaving the contaminated areas were: fogging of goggles, headache caused by tight goggles, swelling and pain of lower limbs caused by tight lace-up of shoe cover, loose frenulum of the mask, difficult breathing caused by mask that was close to the nose wings, and loosening of face screen. Yet, after new protective measures were applied, the rate of discomfort was reduced, resulting in improved efficiency of nursing work.

\section{Shortening the for materials transfer from/to contaminated areas}

Due to the one-way access policy, medical staff cannot re-enter the clean area warehouse after entering the contaminated area. However, the ward holds the limited spare items; thus, additional personnel must be sent to the contaminated area for item transfer. In addition, there were some obstacles in the vision, action and communication of medical personnel under the protection state. Due to the small scope of vision, poor mobility, and the impact of protective equipment on sound production and hearing,Communication will take more time than normal. When there are insufficient items and need to contact the medical staff in clean area to supplement, the ongoing nursing operation will be interrupted and the nurse's time will be occupied [23]. Information communication was conducted on average 2-3 times per shift, and each time occupied 5-20 minutes of a nurse. After applying new measures, the time for transferring items between inside and outside of contaminating area was reduced compared to the control group (1.98 \pm 1.41 vs. $2.52 \pm 1.97)$.

\section{Repeated replenishment of bedside consumables}

The ICU ward is a large open-plan room with double-sided bed layout [24], and the office area between the beds on both sides. In our hospital, the consumables were mainly placed in two cabinets near the 
entrance side and the treatment room. There were five treatment vehicles in the ward, carrying different types and quantity of consumables. Before applying new measures, an average of 7-11 people per shift, each taking 1-5 minutes, were responsible for carrying bedside consumables. After the new measures were applied, the time and number of persons carrying bedside consumables were significantly reduced (4-9 person-times per shift). By establishing the corresponding management system to avoid missing the best rescue opportunity due to lack of materials, the overall emergency response efficiency was improved.

\section{Study Limitations}

The present study has a few limitations. The early nursing experience and improvement measures of the epidemic were limited. Also, the sample size was small.

\section{Conclusion}

In this study, we established and validated a new plan for improving the efficiency of nursing work for patients with severe COVID-19. In a word, the waste of working time can be reduced by improving the wearing comfort of protective clothing, improving the system of material transfer and supplement, and the use of consumables. In conclusion, the clinical work experience explored in practice can improve the efficiency of nursing work and provide a reference for anti-epidemic front-line nurses.

\section{Abbreviations}

novel coronavirus pneumonia (COVID-19)

\section{Declarations}

\section{Ethics approval and consent to participate}

The study was approved by the ethics committee of First Affiliated Hospital of PLA Air Force Military Medical University. All participants provided written informed consent.

\section{Consent for publication}

Not applicable.

\section{Availability of data and materials}

The datasets used and/or analysed during the current study are available from the corresponding author on reasonable request.

\section{Competing interests}


None.

\section{Funding}

None.

\section{Authors' contributions}

LMM, ZY, LM, and WY contributions to conception and design, and analysis and interpretation of data, drafting the article, final approval of the version to be published. LMH contributions to acquisition of data, analysis and interpretation of data, conception and design. ZN contributions to revising it critically for important intellectual content. All of them are in agreement to be accountable for all aspects of the work in ensuring that questions related to the accuracy or integrity of any part of the work are appropriately investigated and resolved.

\section{Acknowledgements}

The authors would like to thank the whole staff of WuHan Huoshenshan hospital, and all patients who participated in this study. LMM, ZY, LM, and WY contributions to conception and design, and analysis and interpretation of data, drafting the article, final approval of the version to be published. LMH contributions to acquisition of data, analysis and interpretation of data, conception and design. ZN contributions to revising it critically for important intellectual content. All of them are in agreement to be accountable for all aspects of the work in ensuring that questions related to the accuracy or integrity of any part of the work are appropriately investigated and resolved.

\section{Conflict of interest}

The authors declare no competing interests.

\section{References}

1. World Health Organizition.Coronavirus disease (COVID-19) Pandemic. https://www.who.int/emergencies/diseases/novel-coronavirus-2019. Accessed 18 July 2020.

2. Professional Committee of Critical Care Medicine CRHA, Youth Committee of Special Committee of Critical Care Medicine, Chinese Research Hospital Association.: Expert consensus on the diagnosis and treatment of severe and severe novel coronavirus pneumonia. Chinese Critical Care Medicine 2020.

3. New Coronavirus Pneumonia Diagnosis and Treatment. Plan (Trial Version Six). Chinese Journal of Infection Control. 2020;19(02):192-5.

4. Li C, Huang X. Expert Consensus on Personal Protection of Work in Different Areas of Medical Institutions during the New Coronary Pneumonia Epidemic. Chinese Journal of Infection Control. 2020;19(03):199-213. 
5. Wang Y, Wu W, Cheng Z, Tan X, Yang Z, Zeng X, Mei B, Ni Z, Wang X. Super-factors associated with transmission of occupational COVID-2019 infection among healthcare staff in Wuhan, China. The Journal of hospital infection 2020.

6. Weihong R, Lihan S, Miaoling Z, Huan Z, Junhua C. Nursing difficulties and countermeasures for 12 critically ill patients with novel coronavirus pneumonia. Journal of Nursing. 2020;27(05):68-71.

7. Kovner C. Policy, Politics, Nursing Practice, and COVID-19. Policy Politics Nurs Pract. 2020;21(2):55.

8. Xiu-jie Z, Tie-ying S, Li S. COVID-19: What is next for nursing in public health emergency? Nurse Education in Practice 2020, 46.

9. Hoogendoorn ME, Margadant CC, Brinkman S, Haringman JJ, Spijkstra JJ, de Keizer NF. Workload scoring systems in the Intensive Care and their ability to quantify the need for nursing time: A systematic literature review. International journal of nursing studies. 2020;101:103408.

10. Prevention CCfDCa: Epidemiology Group of Emergency Response Mechanism of Novel Coronavirus Pneumonia. Epidemiological characteristics of novel coronavirus pneumonia. Chinese Journal of Epidemiology. 2020;41(2):145-51.

11. Li L, Wu A. Discussion on Common Confusion in Prevention and Control of Nosocomial Infection of Novel Coronavirus. Chinese Journal of Infection Control. 2020;19(02):105-8.

12. Huang C, Wang Y, Li X, Ren L, Zhao J, Hu Y, Zhang L, Fan G, Xu J, Gu X, et al. Clinical features of patients infected with 2019 novel coronavirus in Wuhan, China. Lancet. 2020;395(10223):497-506.

13. Chen N, Zhou M, Dong X, Qu J, Gong F, Han Y, Qiu Y, Wang J, Liu Y, Wei Y, et al. Epidemiological and clinical characteristics of 99 cases of 2019 novel coronavirus pneumonia in Wuhan, China: a descriptive study. Lancet. 2020;395(10223):507-13.

14. Yang X, Yu Y, Xu J, Shu H, Xia J, Liu H, Wu Y, Zhang L, Yu Z, Fang M, et al. Clinical course and outcomes of critically ill patients with SARS-CoV-2 pneumonia in Wuhan, China: a single-centered, retrospective, observational study. The Lancet Respiratory medicine. 2020;8(5):475-81.

15. Alhazzani W, Møller MH, Arabi YM, Loeb M, Gong MN, Fan E, Oczkowski S, Levy MM, Derde L, Dzierba A, et al. Surviving Sepsis Campaign: guidelines on the management of critically ill adults with Coronavirus Disease 2019 (COVID-19). Intensive care medicine. 2020;46(5):854-87.

16. Lumley C, Ellis A, Ritchings S, Venes T, Ede J: Using the Systems Engineering Initiative for Patient Safety (SEIPS) model to describe critical care nursing during the SARS-CoV-2 pandemic (2020). Nursing in critical care 2020, 25(4):203-205.

17. Choi KR, Skrine Jeffers K, Cynthia Logsdon M. Nursing and the novel coronavirus: Risks and responsibilities in a global outbreak. Journal of advanced nursing. 2020;76(7):1486-7.

18. Zhong S, Cao X. Implementation and effect of nursing human resources deployment plan under the emergency treatment of new coronavirus pneumonia. Chinese Nursing Management. 2020;20(02):226-9.

19. World Health Organization. Coronavirus Disease (Covid-19) Outbreak: Rights, Roles And Responsibilities Of Health Workers, Including Key Considerations For Occupational Safety And Health. World Health Organization (WHO).(2020). https://www.who.int/docs/default- 
source/coronaviruse/who-rights-roles-respon-hw-covid-19.pdf?sfvrsn=bcabd401_0. Accessed 31 March 2020.

20. World Health Organisation. Modes of transmission of virus causing COVID-19: implications for IPC precaution recommendations. https://www.who.int/newsroom/commentaries/detail/modes-oftransmission-of-virus-causing-covid-19-implications-foripc-precaution-recommendations. Accessed 31 March 2020.

21. Wierenga KL, Moore SE. Adapting to Uncertainty: Nursing Responsiveness to COVID-19. J Cardiovasc Nurs. 2020;35(4):322-3.

22. Xiaodan LL. C: Application and effect observation of different protective eyepiece antifogging methods in new coronary pneumonia intensive care isolation unit. Journal of Nurses Training: 1-3 2020,35(09):817-819., 35(09):817-819.

23. Qiu Y, Ye B. Study on effective training methods of personal protection against new coronary pneumonia. Chinese Journal of Infection Control. 2020;19(06):513-7.

24. Fang H, Zhang Q. Nosocomial infection control and prevention in a specialist hospital for novel coronavirus pneumonia: Taking Huoshenshan Hospital as an example. Mil Med. 2020;44(04):27881. 